\title{
Why Mars Sample Return is a Mission Campaign of Compelling Importance to Planetary Science and Exploration
}

\author{
A White Paper for the Planetary Decadal Survey
}

Primary authors:

H. Y. McSween ${ }^{1}$ (865-386-1912, mcsween@utk.edu), M. M. Grady ${ }^{2}$, Kevin McKeegan ${ }^{3}$, D. W. Beaty $^{4}$, B. L. Carrier ${ }^{4}$

${ }^{1}$ University of Tennessee, ${ }^{2}$ Open University, ${ }^{3}$ University of California, Los Angeles, ${ }^{4} \mathrm{Jet}$ Propulsion Laboratory/California Institute of Technology

Additional signatories:

Name

Adrian Brown

Alberto G. Fairén

Alexandre Anesio

Andrew Czaja

Ann C. Vandaele

Ari Koeppel

Arya Udry

Audrey Bouvier

Barbara Cohen

Benjamin Weiss

Bernard Marty

Brian D. Wade

Briony Horgan

Carlton Allen

Christopher Herd

Corliss Kin I Sio

Dale T. Andersen

Daniel P. Glavin

David Shuster

David T. Flannery

Devanshu Jha

Eleni Ravanis

Elisabeth Hausrath

Elliot Sefton-Nash

Ernst Hauber

Felipe Gomez

Frances Westall

Francis M. McCubbin

Frédéric Moynier

Gerhard Kminek

Gian Gabriele Ori

Gözen Ertem

Guido Kreck
Affiliation

Plancius Research

Centro de Astrobiologia (CSIC-INTA)

Aarhus University, Denmark

University of Cincinnati

Royal Belgian Institute for Space Aeronomy, Belgium

Northern Arizona University

University of Nevada, Las Vegas

Universität Bayreuth, Bayerisches Geoinstitut, Germany

NASA Goddard Space Flight Center

Massachusetts Institute of Technology

CRPG/CNRS \& Université de Lorraine

Michigan State University

Purdue University

NASA Johnson Space Center (retired)

University of Alberta

Lawrence Livermore National Laboratory

The SETI Institute

NASA Goddard Space Flight Center

University of California, Berkeley

Queensland University of Technology

MVJ College of Engineering, Bangalore, India

ESA (ESAC)

University of Nevada, Las Vegas

ESA (ESTEC)

German Aerospace Center (DLR)

Centro de Astrobiología (CSIC-INTA)

CNRS-Orléans, France

NASA Johnson Space Center

Université de Paris, Institut de Physique du Globe de Paris

ESA

IRSPS, Univ d'Annunzio, Italy

The SETI Institute

Fraunhofer Institute for Manufacturing Engineering and 
Harald Reichert

Henner Busemann

Henning Dypvik

Hope Ishii

J Andy Spry

J.R. Skok

Jack D. Farmer

Janice Bishop

Jeffrey R. Johnson

Jennifer L Eigenbrode

John Bridges

John Robert Brucato

Jorge I. Núñez

Jose C. Aponte

Juan M. Madariaga

Juergen Thieme

Juliane Gross

Katherine French

Kathleen Campbell

Kim Tait

Lukas Adam

Lydie Bonal

Mackenzie Day

Mark A. Sephton

Nathalie A. Cabrol

Martin J. Van Kranendonk

Maxwell M. Thiemens

Meenakshi Wadhwa

Michael Velbel

Nicholas Tosca

Nicolas Dauphas

Nicole Schmitz

Pascal Lee

Petra Rettberg

R. Mark Elowitz

Rachel Mackelprang

Roberto Orosei

Sandra Siljeström

Scott M. McLennan

Stephanie C. Werner

Susanne P. Schwenzer

Tilmann Denk

Timothy S. Swindle

Tomohiro Usui
Automation IPA

European Synchrotron Radiation Facility (ESRF), Grenoble,

France

ETH Zurich

University of Oslo, Norway

Hawaii Institute of Geophysics \& Planetology/

University of Hawaii

The SETI Institute

The SETI Institute

Arizona State University

The SETI Institute

Johns Hopkins University Applied Physics Laboratory

NASA Goddard Space Flight Center

University of Leicester, UK

INAF - Astrophysical Observatory of Arcetri, Firenze, Italy

Johns Hopkins University Applied Physics Laboratory

CUA/NASA Goddard Space Flight Center

University of the Basque Country, Bilbao, Spain

National Synchrotron Light Source II,

Brookhaven National Laboratory

Rutgers University, New Jersey

U.S. Geological Survey

University of Auckland, New Zealand

Royal Ontario Museum, Toronto, Ontario, Canada

University of Leicester, UK

Institut de Planétologie et d'Astrophysique de Grenoble,

Université Grenoble Alpes, CNRS CNES, Grenoble France

University of California Los Angeles

Imperial College London, UK

The SETI Institute

University of New South Wales

Laboratoire G-Time, Université Libre de Bruxelles, Belgium

Arizona State University

Michigan State University

University of Oxford, UK

The University of Chicago

German Aerospace Center (DLR)

Mars Institute \& SETI Institute

German Aerospace Center (DLR), Köln, Germany

Recent Open University PhD

California State University Northridge

Istituto Nazionale di Astrofisica, Italy

RISE Research Institutes of Sweden

Stony Brook University

CEED-GEO, University of Oslo, Norway

The Open University, UK

German Aerospace Center (DLR)

Lunar \& Planetary Laboratory, University of Arizona

Institute of Space and Astronautical Science (ISAS),

Japan Aerospace Exploration Agency (JAXA) 


\section{Udo Gommel}

Vinciane Debaille

Yang Liu

Yasmina M. Martos

Yulia Goreva
Fraunhofer Institute for Manufacturing Engineering and Automation IPA

Laboratoire G-Time, Université Libre de Bruxelles, Belgium Jet Propulsion Laboratory/California Institute of Technology NASA Goddard Space Flight Center

Jet Propulsion Laboratory/California Institute of Technology 


\section{BACKGROUND FOR MARS SAMPLE RETURN}

Goals for Mars exploration are to understand Mars as a planet, its role as a potential host for life, and its place within Solar System evolutionary history. An important step in exploration that will lead to significant advances is the return of samples of Mars to Earth.

Mars Sample Return (MSR) is an undertaking of international significance. It is not a single mission, but a series of missions requiring substantial technical and engineering advances underpinned by increased scientific understanding of the planet. MSR will only succeed if it is an international endeavor - a fact that was recognized in 2018, when NASA and ESA signed a Joint Statement of Intent to seek ways to carry out MSR by means of an international partnership. The first mission in the MSR campaign is Mars-2020. This NASA-funded mission, carrying the rover Perseverance, is scheduled to launch in 2020 and will collect and cache samples from Jezero crater. The first follow-up mission, funded by ESA, is intended to launch in 2026, and is designed to fetch the cached samples and deliver them to a vehicle that will carry them into Mars orbit. A third mission will bring the samples to Earth, nominally in 2031. The international nature of MSR is not limited to the missions that will collect and retrieve material from Mars. Once the samples are returned to Earth, they will become the focus of a major international scientific analysis campaign.

The return of samples from the martian surface has been a major goal of the Mars science community for many years, and a landed mission to initiate MSR (now Mars-2020) was identified as one of the highest-priority missions in the last Planetary Decadal Survey. This white paper, focusing on MSR science, advocates for the follow-on missions that will return those samples. Two related white papers, intended to be linked to this one, describe the engineering requirements and planning for those missions, and the planning for a dedicated NASA curation facility for Mars samples and other essential capabilities that need to be realized in this decade to allow MSR in 2031.

In 2017, the International Mars Exploration Working Group (IMEWG) established the international MSR Objectives and Samples Team (iMOST). The charter for iMOST was to reevaluate and update the sample-related science and engineering objectives of an MSR campaign, given the planning established for the Mars-2020 sample-collecting rover. The iMOST team also defined the measurements and the types of samples that can best address the objectives. The full iMOST report is available as an electronic publication of Meteoritics \& Planetary Science (https://onlinelibrary.wiley.com/doi/epdf/10.1111/maps.13242).

MSR will directly and meaningfully contribute to a number of overarching questions of fundamental importance to planetary science and exploration. Examples are:

1. Does extraterrestrial life exist, or has it ever existed in the Solar System? If so, when, where, and what are/were its attributes?

2. How and where did prebiotic organic chemistry occur, and what role did it play in establishing the required chemical context for the origin of life?

3. What is the history of habitable environments on planets in our Solar System beyond Earth?

4. How did the planets form, differentiate, and evolve as geological and geophysical bodies? 
5. Can we use early geologic and geochemical records throughout the Solar System to interpret how planetary growth and dynamics controlled or altered the distribution of planets and planetesimals during its first billion years?

6. How did planetary atmospheres form and evolve, and what are the processes that caused them to end up in such different states today?

7. What are potential environmental hazards to future planetary exploration, and what kinds of in situ resources are available to support human exploration?

Three complementary datasets - orbital remote sensing, in situ measurements from landers and rovers, and analyses of martian meteorites - have addressed aspects of these questions for Mars. However, each dataset is lacking in some way, and the next logical step for Mars exploration is to return samples from a known location (Grady, 2020). Here we briefly consider how each of these compelling questions will be informed by specific science objectives identified for MSR by the iMOST report. We emphasize that, in some cases, MSR has the potential to answer some questions definitively.

\section{MSR'S CONTRIBUTIONS TO DEFINITIVELY ANSWERING THESE IMPORTANT SCIENTIFIC QUESTIONS}

Planetary exploration is guided and motivated by critical questions, as noted above. Samples returned from Mars will provide a much more meaningful way to address these questions than by means of the samples already in hand (martian meteorites), which unfortunately lack geologic context, constitute a biased sampling in terms of lithology and ages, and have commonly been altered by shock during launch from Mars. Two aspects to be considered are (1) how MSR provides more complete and definitive answers to these questions for Mars, and (2) how results for Mars improve understanding of the Solar System.

\section{Does extraterrestrial life exist, or has it ever existed in the Solar System? If so, when, where, and what are/were its attributes?}

This question motivates much of NASA's planetary exploration program; an affirmative answer, besides its intrinsic scientific importance, would have significant societal impact. The challenge is not only to be able to detect relatively obvious life forms, but also to be able to recognize more ambiguous evidence. Search mechanisms must be as complete as possible and able to react to both "maybe" outcomes and "if not, why not" outcomes. Life detection is a process, not just "yes-no" tests. MSR allows us to use the entirety of Earth's laboratory capabilities, optimizing the chances of detecting and correctly interpreting subtle signals. This is especially important for Mars, the one other planet that has had an Earth-like geologic history.

Uniquely establishing evidence for extinct life can be especially challenging, of necessity involving a combination of field and laboratory studies to document habitability, to assess the preservation potential for biosignatures, and to document the absence of contamination (Des Marais et al., 2008; Westall et al., 2015). On Earth, the debate on evidence of past life in our oldest rocks has raged for decades. That research demonstrates the importance of carefully collected, well documented samples with known context. Laboratory investigations require organic extraction protocols and molecular/stable isotopic characterizations, as well as 
microscale observations, that are only possible in terrestrial laboratories (Sephton and Botta, 2005). Complete characterization, never achievable in remote sensing, is critical. For example, a key biosignature is complex organic molecules whose formation and abundances relative to other compounds are unachievable in the absence of life (Cronin and Walker, 2016). For fossilized life, it is also necessary to date the enclosing sample accurately.

For extant life, the requirements for laboratory analysis protocols are even more stringent, requiring careful sample handling that excludes contamination with terrestrial life, as well as documentation of metabolism/respiration and of reproduction in culture experiments. The discovery of life on Mars would allow us to test assumptions about universal requirements for life, possibly uncover novel ways in which life processes are carried out, and identify commonalities and divergencies between life on Earth and Mars. Any definitive discovery of extraterrestrial life, and certainly any understanding of its biological and chemical characteristics, require coordinated tests that can only be accomplished in Earth-based laboratory settings. MSR will permit replication of significant findings, one of the gold standards of science, thereby increasing confidence in answers to this profound question.

\section{How and where did prebiotic organic chemistry occur, and what role did it play in establishing the required chemical context for the origin of life?}

One necessary condition for life's origin (in addition to water) is the availability of appropriate organic compounds. We have very little knowledge of this from Earth, but the ancient geologic record of Mars is better preserved. Refractory abiotic organic carbon has been detected in both meteorites (Steele et al., 2016) and by SAM (Eigenbrode et al., 2018), but analyses of more labile species like amino acids has been an issue due to terrestrial contamination or instrument background. Sample return will allow this inventory of nonrefractory carbon to be analyzed. Samples carefully selected by rover team scientists on the basis of their potential to acquire and preserve intact organic matter, as opposed to random, biased samples launched as meteorites, allow a much better chance of understanding the full complexity of prebiotic chemistry. By analyzing the molecular structures (including enantiomorphs) and compound-specific isotopic compositions of organic compounds, it may be possible to determine the precursors and reaction mechanisms that produced the molecules and the conditions under which they formed, and to constrain the locations in the cosmos for organic synthesis. Comparison of results from Mars rocks with primitive meteorites and cosmic dust may provide information on the role of prebiotic chemistry throughout the Solar System.

\section{What is the history of habitable environments on planets in our Solar System beyond Earth?}

Five geologic environments are presently recognized as potentially habitable and capable of preserving evidence of habitation (Hays et al., 2017): sedimentary systems; hydrothermal systems; deep groundwater systems; locations that experienced water/rock/atmosphere interactions; and iron-rich springs. All these environments involve water. Jezero crater, the Mars-2020 landing site, exposes a partially eroded, ancient sedimentary system of lacustrine and deltaic deposits (Gouge et al., 2018) and thus qualifies as a potentially habitable environment. The Mars-2020 rover will address how the environmental conditions arose and evolved with time, but essential supporting evidence will come from the returned samples. Analyses by instruments only available in terrestrial laboratories will allow the detailed organic, stable 
isotopic, petrologic, and geochronologic studies necessary to definitively establish the conditions that led to onset of habitability, as well as its duration.

\section{How did the planets form, differentiate and evolve as geological and geophysical bodies?}

At present, much of what we surmise about Mars' geologic evolution comes from martian igneous meteorites, which are not fully representative of the crust. All of them are mafic rocks or ultramafic cumulates, most are less than a few hundred million years old (rocks billions of years old dominates the crust of Mars), and many have been highly altered by shock metamorphism (McSween, 2015; Filiberto, 2017). Studies of volcanic rocks from a few martian sites by rovers indicate that the meteorites provide a very limited and biased view of Mars igneous rock compositions (e.g. Sautter et al., 2016).

MSR from Jezero crater will certainly include sedimentary rocks whose stratigraphy, texture, mineralogy, and geochemistry can provide information on provenance, petrologic diversity, absolute ages, history of water, and evolution of environmental conditions and climate. Jezero formed on the edge of the $4.0 \mathrm{Ga}$ Isidis basin, and the rover may sample exposed basement rocks, which include megabreccias (observed from orbit) with diverse clasts. A mafic capping unit in the crater may be a lava flow or volcanic sediments. In any case, explorations by all Mars rovers to date indicate that igneous rocks are ubiquitous on Mars, either as boulders redistributed by gravity or flowing water, or as ejecta from large impacts. The ability to select samples that are relatively unaffected by shock, that are older than Amazonian, that may have experienced significant fractionation, or that are breccias with diverse lithologies, would provide major advances to our understanding of martian petrogenesis. Returned igneous rocks, especially of Noachian or Hesperian age, would provide a thermochemical record of the planet not presently available and serve as a new window on the nature of its interior. Their trace element abundances and radiogenic isotopic compositions will allow insights into the differentiation of the mantle and core, as well as address fundamental questions about the evolution of martian magmatism through time. As demonstrated by martian meteorites, some isotope chronometers can be disturbed by subsequent geologic processes, so reliability must be established by concordant ages from multiple isotopic systems, which can only be accomplished in Earth-based laboratories. Radiogenic isotope studies can constrain the evolutionary timeline for the planet and, if samples can be tied to specific geologic units with determined relative ages, serve as calibration points for assigning absolute ages to crater-density distributions. Although Mars does not have an active core dynamo today, paleomagnetic studies of one ancient martian meteorite (Weiss et al., 2008) indicate an active dynamo that shut down at $\sim 4.0 \mathrm{Ga}$ (another aspect of interest in the Jezero site). Paleomagnetism studies of carefully selected, oriented samples in Earth-based laboratories can reveal the history of the planetary dynamo, constrain the present model of mantle convection and possible early existence of plate tectonics, and test the hypothesis that loss of the dynamo led to atmospheric stripping by the solar wind and subsequent climate change.

\section{Can we use early geologic and geochemical records throughout the Solar System to interpret how planetary growth and dynamics controlled or altered the distribution of planets and planetesimals during its first billion years?}


Nucleosynthetic stable isotope anomalies $\left({ }^{53} \mathrm{Cr},{ }^{50} \mathrm{Ti},{ }^{95} \mathrm{Mo}\right.$, etc.) in meteorites indicate two distinct isotopic reservoirs in the early Solar System (Scott et al., 2018), hypothesized to have been separated in the nebula by the rapid growth of proto-Jupiter. Although these isotopes have been measured in young martian meteorites, we do not have comparable analyses of ancient martian samples that would be provided by MSR.

Instabilities in the motions of the giant planets in the first billion years may have scattered planetesimals, thus affecting the collision/impact records of planets (e.g. Morbidelli et al., 2012). The evidence for such scattering comes mainly from the impact record inferred from analyses of the Apollo lunar samples. However, there are major questions about the timing and duration of large impacts in the inner Solar System, suggesting that the "Late Heavy Bombardment" may not have been a spiked or singular event (Bottke and Norman, 2017) or even it existed at all. The absolute chronology of some impact events on Mars can potentially be quantified by radiogenic isotope analyses of selected returned samples. Any information on early collisions at Mars' orbital position would help us understand the dynamics of Solar System bodies as a whole.

\section{How did planetary atmospheres form and evolve, and what are the processes that caused them to end up in such different states today?}

A decades-long debate about the compositional evolution of the martian atmosphere still remains unresolved (Ott, 1988; Schwenzer et al., 2016). MSR will collect and return a significant sample of the modern martian atmosphere (both headspace gas in collection tubes and a proposed dedicated gas sample), and solid samples will contain trapped ancient gases. Although young martian meteorites provide trapped atmosphere from the last few hundred million years (based on their young shock ages), we have no samples of the ancient atmosphere. Certain secondary minerals can also equilibrate with the atmosphere and thus be used to infer its composition. Major and trace elements (including noble gases) and isotopic analyses can provide important constraints on atmospheric origin and evolution (Swindle, 2002), with implications for climate. Although the modern atmosphere has been analyzed remotely to a certain level of precision, the ancient atmosphere can only be extracted and analyzed from rocks in Earth-based laboratories. Understanding how the atmosphere has evolved is critical to constraining the martian volatile inventory and discerning how volatile species have interacted over time with the planet as a geologic system. This information will play a significant role in comparative studies of other planets with atmospheres, such as Earth, Venus, and exoplanets.

\section{What are potential environmental hazards to future planetary exploration, and what kinds of in situ resources are available to support human exploration?}

Dust, regolith, and atmosphere samples can define an array of potential health risks in the martian environment and prompt strategies to mitigate them. Knowledge gaps that must be addressed before human missions are possible require the thorough characterization of particulate and gas samples for toxicology, potential chemical and biologic hazards, and engineering assessments of corrosion, electrical effects, maneuverability, and structural integrity (Kminek et al., 2018; Spry et al., 2018).

Returned samples can also allow quantification studies of natural resources that might be used to obtain water and produce fuel, and for building materials, radiation shields, and agriculture for future human exploration (e.g. Portree, 2011). 
Returned samples are also critical for developing appropriate planetary protection protocols for both Mars and Earth. "Breaking the chain of contact" when leaving Mars is technically achievable for robotic missions, but it is not possible for a crewed mission and potential biological hazards must be determined before humans go to Mars.

\section{CONCLUSIONS}

NASA (and ESA) have already made significant investments in an MSR program, and technical planning for the follow-on missions that will retrieve and return to Earth samples cached by Mars-2020 is moving apace. The samples cached will be carefully chosen to address the science objectives discussed above. In terrestrial laboratories the samples will be processed and studied with instruments that have analytical sensitivity and spatial resolution that cannot be matched by remote-sensing techniques. The data obtained have high likelihood of providing definitive answers to questions critical to planetary science. MSR is also essential before human exploration, not only for astronaut safety, but also because once humans go to Mars, the planet can no longer be considered biologically pristine, even with stringent anti-contamination protocols. And like the samples collected by Apollo astronauts, returned samples from Mars are expected to be a very important resource for scientific research for many decades to come, taking advantage of future advances in analytical techniques and knowledge. Moreover, the findings from laboratory analyses of samples have been shown many times to complement and substantially enhance those from remote sensing.

The potential scientific value of MSR has been discussed in the scientific advisory literature for four decades, and the missions have strong international support and participation. Although the answers to the questions posed here are Mars-specific, the questions are of universal importance, and MSR should allow new insights into phenomena and processes that may not be unique to any one planet.

The samples will be from a single collection site, likely collected over traverses of tens of kilometers. Admittedly, trying to constrain the geology of an entire planet using rocks and soils from a single site conveys a certain hubris, but terrestrial geology demonstrates that well-chosen type localities provide data of global significance. The chosen landing site, interpreted as a Noachian paleolake within a crater in petrologically complex basement terrain (e.g. Goudge et al., 2018), represents a critical time interval that is thought to be particularly important from an environmental and possibly biologic perspective - and from a time interval that is not represented in martian meteorites. Moreover, the returned dust and regolith samples will also be used to address knowledge gaps that must be filled before humans explore Mars directly.

It is important that MSR be addressed in this Planetary Decadal Survey, because planning for certain things such as sample curation must occur in the 2020's and slipping from a 2031 timeline would require different engineering solutions than are presently available (described more fully in companion white papers). MSR is of compelling importance to planetary science and exploration. It's time to finish what we started.

\section{References Cited}

Bottke W. F. and Norman M. D. (2017) The Late Heavy Bombardment. Ann. Rev. Earth Planet. Sci., 45, 619-647. 
Cronin L. and Walker S. I. (2016) Beyond prebiotic chemistry. Science, 352, 1174-1175.

Des Marais D. J. et al. (2008) The NASA Astrobiology roadmap. Astrobiol., 8, 715-730.

Eigenbrode J. L. et al. (2018) Organic matter preserved in 3-billion-year-old mudstones at Gale crater, Mars. Science, 360, 1096-1101.

Filiberto J. (2017) Geochemistry of Martian basalts with constraints on magma genesis. Chem. Geol., 466, 1-14.

Gouge T. A. et al. (2018) Stratigraphy and paleohydrology of delta channel deposits in Jezero crater, Mars. Icarus, 301, 58-75.

Grady M. (2020) Exploring Mars with returned samples. Space Sci. Rev., in press.

Hays L. E. et al. (2017) Biosignature preservation and detection in Mars analog environments. Astrobiol., 17, 363-400.

Kminek G. et al. (2018) Report on the COSPAR workshop on refining planetary protection requirements for human missions.

McSween H. Y. (2015) Petrology on Mars. Amer. Mineral., 100, 2380-2395.

Morbidelli A. et al. (2012) Building terrestrial planets. Ann. Rev. Earth Planet. Sci., 40, 251-275.

Ott U. (1988) Noble gases in SNC meteorites. Geochim. Cosmochim. Acta, 52, 1937-1948.

Portree D. S. F. (2011) Humans to Mars: Fifty years of mission planning. https://history.nasa.gov/monograph21.pdf.

Sautter V. et al. (2016) Magmatic complexity on early Mars as seen through orbital, in situ and meteorite data. Lithos, 254, 36-52.

Schwenzer S. P. et al. (2016) Fractionated noble gases in the nakhlite meteorites. In Developments in Noble Gas Understanding and Expertise, CRPG, 13-15.

Sephton M. A. and Botta O. (2005) Recognizing life in the Solar System: guidance from meteoritic organic matter. Intern. J. Astrobiol., 4, 269.

Scott E. R. D. et al. (2018) Isotopic dichotomy among meteorites and its bearing on the protoplanetary disk. Astrophys. J., 854, 164.

Spry J. A. et al. (2018) Planetary protection knowledge gapsfor future Mars human missions. $48^{\text {th }}$ Intern. Conf. Environ. Systems, https://ttu-ir.tdl.org/ttu-ir/handle/2346/74252.

Steele A. et al. (2016) The provenance, formation and implications of reduced carbon phases in martian meteorites. Meteorit. Planet. Sci., 51, 2203-2225.

Swindle T. D. (2002) Martian noble gases. Rev. Mineral. Geochem., 47, 171-190.

Weiss B. P. et al. (2008) Paleointensity of the ancient Martian Magnetic field. Geophys. Res. Lett., 35, L23207.

Westall F. et al. (2015) A hydrothermal-sedimentary context for the origin of life. Astrobiol., 18, 259-293. 